Med Klin Intensivmed Notfmed 2013 .

108:622-623

DOI 10.1007/s00063-013-0255-x

Online publiziert: 15 . November 2013

(c) Springer-Verlag Berlin Heidelberg 2013

H. Messmann ${ }^{1} \cdot$ C. Trautwein ${ }^{2}$

${ }^{1}$ III. Medizinische Klinik, Klinikum Augsburg

2 Medizinische Klinik III, Universitätsklinikum Aachen, AÖR, Aachen

\title{
Der gastroenterologische Intensivpatient - ein besonderer Problempatient?
}

Die Gastroenterologie nimmt einen festen Platz in der Intensivmedizin ein. Dabei kommen der Fachdisziplin verschiedene Aufgaben zu. Dies betrifft sowohl die interdisziplinäre Behandlung von Krankheitsbildern, die primär originär nicht aus dem Fachgebiet kommen, aber auch fachspezifische Krankheitsbilder, die Ärztinnen bzw. Ärzte der Gastroenterologie federführend betreuen sollten. Im aktuellen Themenheft haben wir daher 5 wichtige Krankheitsbilder der Gastroenterologie ausgewählt, denen Sie häufig auf der Intensivstation begegnen und die dann Ihre spezifische Expertise erfordern.

In den letzten Jahren kam es zu einem dramatischen Anstieg von Clostridiumdifficile-Infektionen. Neben dem rasanten Anstieg des Krankheitsbilds finden sich immer mehr schwerwiegende und lebensbedrohliche Verläufe. Gerade multimorbide und ältere Patienten sind von dieser Erkrankung in besonderem Maße betroffen. Herr Ebigbo gibt Ihnen mit seinem Beitrag einen Überblick aber auch Ausblick darüber, wie wir mit diesem Krankheitsbild umgehen sollten.

Akute gastrointestinale Blutungen stellen schon immer eine Herausforderung für den Intensivmediziner dar. Trotz verbesserter endoskopischer Möglichkeiten - sowohl diagnostisch wie therapeutisch - liegt die Mortalität seit Jahren unverändert bei 5-10\%. Dies liegt zum einen an unserer zunehmend älter werdenden Gesellschaft aber auch an den mit den Blutungen einhergehenden Begleiterkrankungen. Beide Faktoren - Alter und Komorbidität
- sind im Rockall-Score mit abgebildet und klassifizieren das Mortalitäts- und Reblutungsrisiko. Ein anderer bedeutender Faktor, der den Verlauf von akuten Blutungen wesentlich beeinflusst, ist die medikamentöse Therapie insbesondere bei kardiologischen Patienten. Der Einsatz von Antikoagulanzien aber auch Thrombozytenaggregationshemmern ist durch neue kardiologische Interventionsverfahren enorm gestiegen und damit auch die Häufigkeit und Schwere von gastrointestinalen Blutungen. Herr Braun diskutiert in seinem Artikel das Problemfeld Antikoagulanzien/Thrombozytenaggregationshemmer und gastrointestinale Blutung mit einem besonderen Blick auf die sog. neuen oralen Antikoagulanzien (NOAK).

Das abdominelle Kompartmentsyndrom, bislang ein Krankheitsbild, das v. a. die operative Intensivmedizin beschäftigt, wird zunehmend auch für die konservative Intensivmedizin interessant. Das rechtzeitige Erkennen von Risikopatienten und v. a. das daran Denken ist eines der Hauptbotschaften in dem Beitrag von Reibetanz. Das Monitoring dieses Krankheitsbilds durch Messen des Blasendrucks ist denkbar einfach und sollte fester Bestandteil einer jeden konservativen Intensivstation sein. Die Therapie hingegen ist oftmals schwierig und interdisziplinär. Fehlt eine kausale Therapie, dann sind symptomatische Maßnahmen zur Senkung des intraabdominellen Drucks oft lebensrettend.

Die Prognose von Patienten mit Leberzirrhose bei bedrohlichen Komplikationen hat sich in den letzten Jah- 
ren verbessert, da durch die frühzeitige Diagnose und gezielte Therapie letale Komplikationen verhindert werden können. Dies gilt sowohl für die akute Varizenblutung als auch für die spontan bakterielle Peritonitis oder das hepatorenale Syndrom. Im Artikel von Herrn Lerchmacher et al. werden diese Themen aufgearbeitet und das Vorgehen in diesen Situationen detailliert besprochen.

Das akute Leberversagen stellt ein dramatisches Krankheitsbild dar. Diese Patienten müssen daher frühzeitig in ein Lebertransplantationszentrum verlegt werden, um diese Therapieoption verfügbar zu haben. Ein hoher Anteil dieser Patient stirbt oder kann nur durch den Organersatz gerettet werden. Im Beitrag von Herrn Streetz et al. werden daher das gezielte Vorgehen und die Therapie bei akutem Leberversagen dargestellt.

Wir hoffen, dass wir Ihnen mit dieser Auswahl einen guten Überblick über wichtige Themengebiete der gastroenterologischen Intensivmedizin vermitteln können und Sie dadurch viele praktische Hinweise für Ihre tägliche Praxis erhalten.

Mit herzlichen Grüßen

Ihre

Helmat ly sumer

Prof. Dr. Helmut Messmann

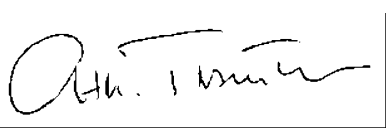

Prof. Dr. Christian Trautwein

\section{Korrespondenzadressen}

Prof. Dr. H. Messmann
III. Medizinische Klinik,
Klinikum Augsburg
Stenglinstrasse 2,
86156 Augsburg
helmut.messmann@
klinikum-augsburg.de

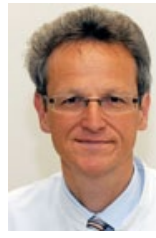

Prof. Dr. C. Trautwein Medizinische Klinik III, Universitätsklinikum Aachen, AÖR

Pauwelsstraße 30, 52074 Aachen ctrautwein@ukaachen.de

\title{
Hier steht eine Anzeige.
}

\author{
Springer
}

